\title{
Particularites Diagnostiques Et Pronostiques De La Dissection Aortique Au Cnhu-Hkm De Cotonou, Benin : A Propos De 6 Cas En 2017
}

Adjagba Philippe Mahouna, MD, Cardiologue, Assistant Chef de Clinique

Bognon Rosaire, MD, Cardiologue

Clinique Universitaire de Cardiologie, CNHU-HKM, Cotonou,

Université d'Abomey-Calavi (UAC), Bénin

Sonou Arnaud, MD, Cardiologue, Assistant Chef de

\section{Clinique}

Centre Hospitalier Universitaire Départemental de l'Ouémé et du Plateau, Porto-Novo, Université d'Abomey-Calavi (UAC), Bénin

Hounkponou Murielle, MD, Cardiologue, Assistant Chef de Clinique

Djoh Ingrid, $M D$

Assani Moutaïrou Salimatou, MD, Cardiologue

Yessoufou Tchabi, MD, Cardiologue, Maître-AssistantCAMES

Houénassi Martin Dèdonougbo, MD, Cardiologue, Professeur Titulaire-CAMES

Clinique Universitaire de Cardiologie, CNHU-HKM, Cotonou,

Université d'Abomey-Calavi (UAC), Bénin

Doi: 10.19044/esj.2018.v14n18p206 URL:http://dx.doi.org/10.19044/esj.2018.v14n18p206

Abstract

Aortic dissection (DA) defined by longitudinal cleavage of the aortic wall, at the level of the media from an intimal breach, is a diagnostic and therapeutic emergency. Out of a total of 467 hospitalized patients, we reported 6 cases of aortic dissection (DA) observed at CNHU-HKM, Cotonou, in 2017. The patients were 53.33 years old (33-65 years old), and they comprised of 4 women and 2 men. Hypertension was present in 5 patients. Chest pain, the most common telltale sign, was found in 5 patients but typical only in 2 cases. Three (3) patients had severe hypertension when they were admitted, and 2 had a differential arterial pressure of at least $20 \mathrm{mmHg}$ between the arms. The breath of aortic insufficiency was present in 4 patients. Chest X-ray has 
contributed to the diagnosis in the 4 cases where it was prescribed. Cardiac Döppler ultrasonography was perfomed in 5 patients, while the ECG gave no contribution to the diagnosis. The diagnostic confirmation was made by the aortic CT scan in all patients and the AD was type A in 5 cases and type B in 1 case according to the Stanford classification. All patients received medical treatment and only 1 patient received both medical treatment and surgery. The outcome was fatal in 5 patients: death of the patient with type B dissection and 4 deaths out of 5 for type A.

Keywords: Aortic dissection, diagnosis, outcome, Cotonou

\section{Résumé}

La dissection aortique (DA) définie par le clivage longitudinal de la paroi aortique au niveau de la média à partir d'une brèche intimale est une urgence diagnostique et thérapeutique. Nous rapportons 6 cas de dissection aortique (DA) observés au CNHU-HKM de Cotonou en 2017, sur un total de 467 patients hospitalisés. Les patients étaient en moyenne âgés de 53,33 ans (33-65 ans) dont 4 femmes et 2 hommes. L'HTA (Hyper Tension Artérielle) était présente chez 5 patients. La douleur thoracique, signe révélateur le plus fréquent, était retrouvée chez 5 patients mais la douleur typique seulement dans 2 cas. Trois (3) patients avaient à l'admission, une HTA (Hyper Tension Artérielle) de grade 3, et 2 avaient une différentielle d'au moins $20 \mathrm{mmHg}$ entre les bras. Le souffle d'insuffisance aortique était présent chez 4 patients. La radiographie thoracique était contributive au diagnostic dans les 4 cas où elle a été prescrite; l'échographie Döppler cardiaque l'était chez 5 patients alors que 1'ECG (électrocardiogramme) n'a été d'aucune contribution au diagnostic. La confirmation diagnostique a été faite par 1'angioscanner de l'aorte chez tous les patients et la DA était de type A dans 5 cas et de type B dans 1 cas selon la classification de Stanford. Tous les patients ont bénéficié d'un traitement médical et 1 patient d'un traitement médical et d'une chirurgie. L'évolution a été fatale chez 5 patients : décès du patient porteur de la dissection de type $B$ et 4 décès sur 5 pour la DA de type $A$.

Mots-clés: Dissection aortique, diagnostic, évolution, Cotonou

\section{Introduction}

La dissection aortique (DA) se définit par le clivage longitudinal de la paroi aortique au niveau de la média à partir d'une brèche intimale favorisant l'irruption du sang sous pression dans la partie clivée et son extension en aval (Roudaut et al., 2009). Sa prévalence, autrefois faible en Afrique, est en progression parallèlement à l'acquisition et à l'amélioration des outils diagnostics (Sarr et al., 2004). Son diagnostic cliniquement suspecté repose 
essentiellement sur l'imagerie, notamment l'échographie et la tomodensitométrie (TDM). Il s'agit d'une urgence médicochirurgicale difficile à prendre en charge dans les pays à plateau technique limité. Le présent travail se propose, à partir de l'analyse de 6 cas de dissection aortique observés à Cotonou, de dégager les aspects cliniques, paracliniques et pronostiques de la maladie.

\section{Texte principal}

\section{Observations}

$\mathrm{Au}$ cours de l'année 2017, 6 patients ont été hospitalisés pour dissection aortique sur 467 patients hospitalisés, soit une fréquence hospitalière de $1,28 \%$.

\section{Cas 1}

Il s'agit d'une patiente noire âgée de 33 ans, adressée pour la prise en charge d'une douleur thoracique aiguë. Elle ne présentait aucun antécédent particulier. L'interrogatoire retrouvait une notion d'hémoptysie de petite abondance associée à des douleurs latéro-thoraciques type point de côté majorées à l'inspiration profonde et irradiant peu. Par ailleurs, la patiente aurait signalé une vive douleur thoracique prolongée pendant l'accouchement 3 mois auparavant. Elle avait une taille de $1,78 \mathrm{~m}$ pour un poids de $98 \mathrm{~kg}$ soit un IMC (indice de masse corporelle) de 33,91 kg/m2. L'examen physique à l'entrée mettait en évidence un bon état hémodynamique avec une PA (presion arterielle) à 137/83 mmHg, une polypnée avec FR à 29cpm et une désaturation en oxygène à $92 \%$. A l'auscultation cardiaque il y avait un souffle d'insuffisance aortique. On retrouvait une condensation pulmonaire basale droite à l'examen pleuropulmonaire et une hépatalgie sans hépatomégalie à l'examen hépato-digestif. Elle présentait une hyperlaxité ligamentaire. L'examen ophtalmologique était normal. Devant cette hémoptysie avec dyspnée associées aux signes d'insuffisance cardiaque droite (hépatalgie avec reflux hépatojugulaire), une radiographie pulmonaire a été demandée. On objectivait à la radio une cardiomégalie avec ICT (index cardio-thoracique) à 0.72, un élargissement du médiastin et un syndrome interstitiel basal droit. L'échographie doppler cardiaque a objectivé une dilatation anévrismale de l'aorte initiale à $85 \mathrm{~mm}$ avec présence d'un flap intimal et d'une insuffisance aortique grade IV. Il n'y avait pas de bicuspidie. Il n'y avait pas de dysfonction ventriculaire gauche. Un angioscanner thoraco-abdominal a permis d'objectiver: une embolie pulmonaire lobaire supérieure et inférieure bilatérale sans signes de gravité paraclinique, une dilatation anévrismale de la racine aortique et un flap intimal allant de la racine à la crosse aortique compatible avec une dissection aortique type A de Stanford. Le diagnostic de dissection aortique chronique associée à une embolie pulmonaire bilatérale est 
posé. Elle a reçu avec succès un traitement associant hypotenseur et anxiolytique. Aucun traitement anticoagulant n'a été institué. La patiente est décédée un mois après sa sortie d'hospitalisation.

\section{Cas 2}

Il s'agit d'une patiente noire, âgée de 65 ans, adressée en hospitalisation cardiologique pour la prise en charge d'une dissection aortique de découverte scannographique fortuite. Elle avait essentiellement comme antécédent une hypertension artérielle découverte depuis 3 ans, mal suivie.

L'histoire remontait à six (06) semaines plus tôt; une toux grasse avec expectorations blanchâtres ayant nécessité la réalisation d'une radiographie pulmonaire. Devant un élargissement du médiastin objectivé à ladite radiographie, un angioscanner thoracique a été réalisé. Il fut découvert au scanner une dissection aortique de l'aorte ascendante. Il n'y avait pas de douleur thoracique ni de dyspnée. L'examen physique à l'entrée permettait de retrouver une taille de $1,52 \mathrm{~m}$, un poids de $71 \mathrm{~kg}$ soit un IMC de $30,73 \mathrm{~kg} / \mathrm{m} 2$, une hypertension artérielle grade 3 de l'OMS (Organisation Mondiale de la Santé) (180/82 mmHg) et un souffle d'insuffisance aortique $3 / 6$ à l'auscultation cardiaque. L'échographie cardiaque mettait en évidence une aorte initiale dilatée à $65 \mathrm{~mm}$ avec une insuffisance aortique modérée. Le diagnostic retenu était une dissection aortique type A de Stanford et la patiente a bénéficié d'un traitement composé de ramipril 10mg /jr, hydrochlorothiazide $12,5 \mathrm{mg} / \mathrm{jr}$ et d'aténolol $50 \mathrm{mg} / \mathrm{jr}$. L'objectif tensionnel a été atteint au bout de 5 jours de traitement. La patiente a été proposée pour une évacuation sanitaire vers un centre disposant d'une unité de chirurgie cardiovasculaire.

\section{Cas 3}

Il s'agit d'une femme noire, âgée de 50 ans, admise pour une poussée hypertensive associée à des douleurs thoraciques. L'interrogatoire retrouvait une hypertension artérielle découverte depuis cinq ans, irrégulièrement suivie. Elle relatait la survenue quatre (04) jours plus tôt d'une brûlure rétrosternale de durée prolongée, d'intensité moyenne, sans irradiation, aggravée par le décubitus dorsal et calmée par le décubitus latéral. Par ailleurs, elle a présenté un malaise à type de lipothymie. L'examen physique à l'entrée retrouvait une taille de $1,58 \mathrm{~m}$ pour un poids de $85 \mathrm{~kg}$ soit un IMC de $34,05 \mathrm{~kg} / \mathrm{m} 2$, une hypertension artérielle grade 3 de l'OMS $(220 / 130 \mathrm{mmHg})$ avec une anisotension systolique de $20 \mathrm{mmHg}$ en faveur du membre thoracique gauche. L'électrocardiogramme mettait en évidence un rythme jonctionnel accéléré à $63 / \mathrm{mn}$, une hypertrophie ventriculaire gauche et des anomalies de la repolarisation à type d'ondes $\mathrm{T}$ négatives en antérieur étendu et en inférieur. Le télécoeur de face retrouvait une cardiomégalie avec un élargissement du médiastin. A l'échocardiographie trans thoracique on notait une hypertrophie 
concentrique du ventricule gauche, une dilatation de l'aorte ascendante à 45 $\mathrm{mm}$ et un minime décollement péricardique circonférentiel. Le diagnostic de dissection aortique type A de Stanford compliqué d'hémopéricarde a été évoqué et confirmé sur la base de l'angioscanner thoracique. Elle fut mise sous nicardipine en intraveineuse relayée par de l'amlodipine $10 \mathrm{mg} / \mathrm{jr}$, ramipril $5 \mathrm{mg} / \mathrm{jr}$, aténolol $100 \mathrm{mg} / \mathrm{jr}$. Une procédure d'évacuation sanitaire vers un centre spécialisé a été initiée mais elle décéda deux jours plus tard.

\section{Cas 4}

Il s'agit d'un homme noir âgé de 50 ans, hypertendu depuis cinq (05) ans et irrégulièrement suivi. L'interrogatoire retrouvait la survenue sept (07) heures plus tôt d'une précordialgie à type de tiraillement, d'intensité d'emblée maximale réveillant le patient, irradiant dans le dos et les lombes et sans facteur sédatif. L'examen cardiovasculaire physique retrouvait : une taille de $1,63 \mathrm{~m}$ pour un poids de $80 \mathrm{~kg}$ soit un IMC de $30,11 \mathrm{~kg} / \mathrm{m} 2$, une HTA grade 3 de l'OMS (201/105mmHg), un souffle d'insuffisance aortique 3/6. L'électrocardiogramme à l'entrée s'inscrivait en un rythme sinusal régulier à $68 / \mathrm{mn}$ avec une surcharge cavitaire gauche et des anomalies de la repolarisation à type d'ondes T négatives et de sous- décalage du segment ST de $1,5 \mathrm{~mm}$ en antéro-septo-apical. Une échocardiographie transthoracique réalisée préalablement avait retrouvé une dilatation de l'aorte initiale à $44 \mathrm{~mm}$ avec une insuffisance aortique sévère aussi bien que la présence d'un flap intimal faisant évoquer une dissection aortique. Un angioscanner thoracique fut alors réalisé en urgence et conclut sur une dissection aortique s'étendant depuis l'origine de l'aorte jusqu'en dessous des artères rénales avec une absence d'opacification du rein gauche (figures 1 et 2). Le traitement médical associait de la morphine, de la nicardipine et de l'aténolol 100mg/24h. L'évolution sous traitement était marquée par la persistance de la pression artérielle élevée jusqu'au $3^{\text {ème }}$ jour motivant l'adjonction de rilménidine $2 \mathrm{mg} / 24 \mathrm{~h}$ sans succès. Une oligo-anurie s'était installée avec une diurèse à $200 \mathrm{cc}$ et aggravation progressive de la fonction rénale avec une clairance de la créatinine passant de 8,22 à $6,91 \mathrm{ml} / \mathrm{mn}$ (selon l'équation MDRD (Modification of Diet in Renal Disease)). Au $4{ }^{\text {ème }}$ jour d'hospitalisation le patient a présenté un épanchement péricardique circonférentiel de $12 \mathrm{~mm}$. Un œdème aigu pulmonaire réfractaire sous furosémide et risordan est apparu au $5^{\text {ème }}$ jour posant l'indication d'une dialyse en urgence. Au 7ème jour d'hospitalisation, le patient décéda dans un tableau de tamponnade par rupture de l'aorte confirmée face à la majoration de l'épanchement péricardique circonférentiel passant à $26 \mathrm{~mm}$. 


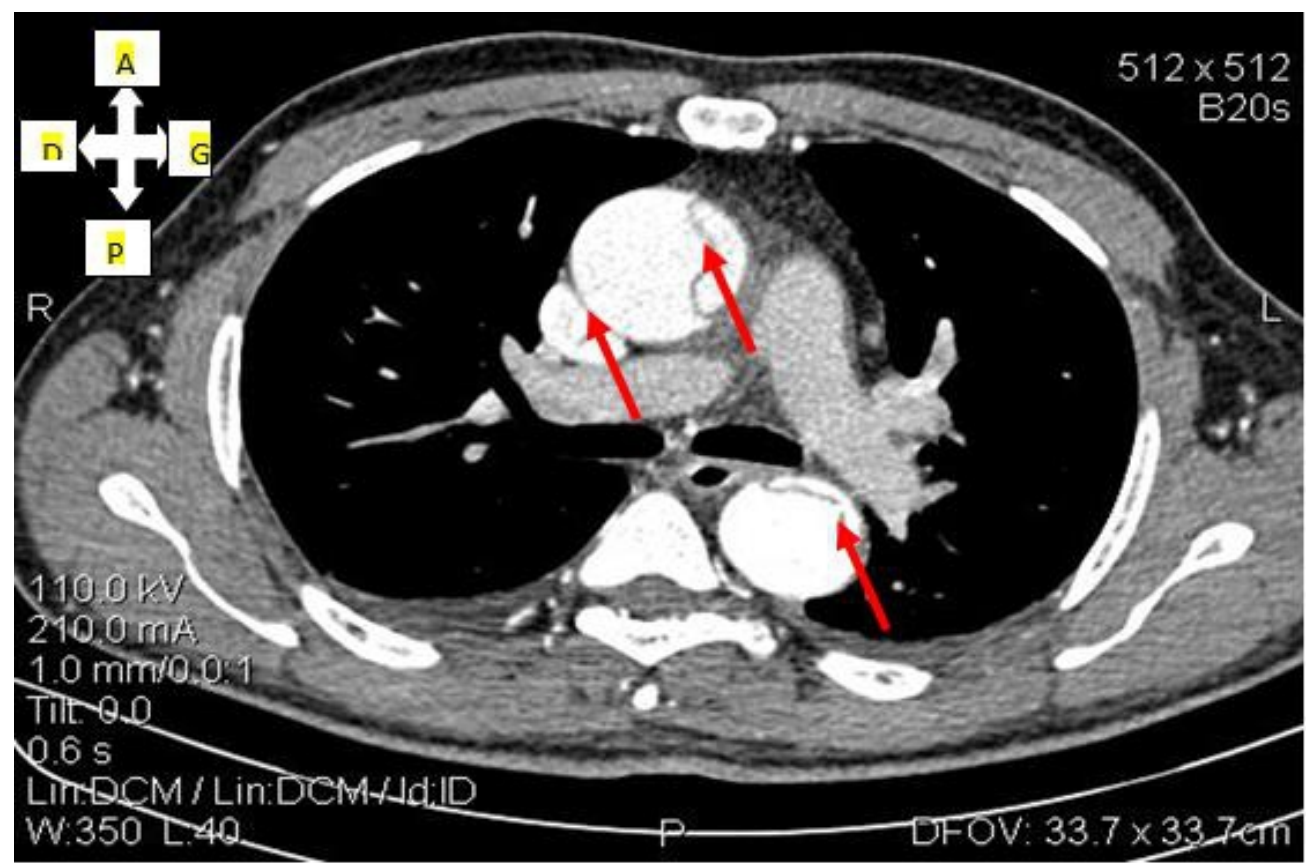

Figure 1. Tomodensitométrie thoracique, coupe axiale, patient de sexe masculin avec dissection aortique de type A de Stanford. Dilatation de l'aorte thoracique avec présence de flaps intimaux (flèches). A (antérieur); P (postérieur); D (droite); G (gauche)

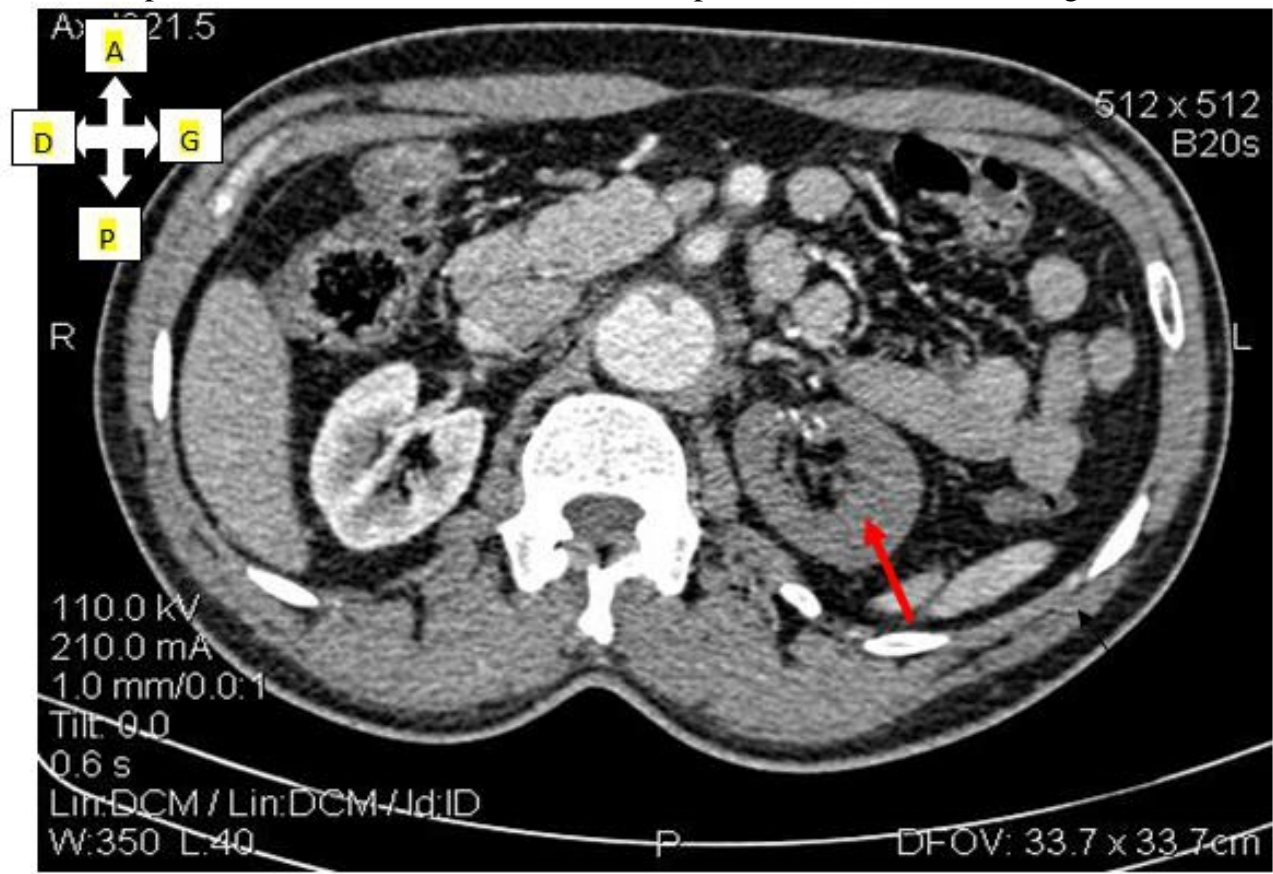

Figure 2. Tomodensitométrie abdominale, coupe axiale, patient de sexe masculin avec dissection aortique de type A de Stanford. Thrombose de l'artère rénale gauche, absence d'opacification du rein gauche (flèche). A (antérieur); P (postérieur); D (droite); G (gauche). 


\section{Cas 5}

Il s'agit d'une femme noire âgée de 57 ans, hypertendue depuis vingt (20) ans; régulièrement suivie; qui a été adressée au service de cardiologie pour la prise en charge d'une dissection aortique de découverte scannographique fortuite. L'interrogatoire retrouvait la survenue, trois (03) mois plus tôt, d'une douleur médio thoracique brutale, vive, d'emblée maximale, ayant durée toute la journée. La patiente ne consulta que 2 semaines plus tard pour asthénie importante associée à une dyspnée. Devant une suspicion d'embolie pulmonaire, un angioscanner thoracique était réalisé. Au scanner, il y avait une polysérite faite de pleurésie, d'ascite et d'épanchement péricardique avec un syndrome alvéolaire paracardiaque bilatéral et un anévrysme de l'aorte ascendante contenant des flaps intimaux méconnus. Elle fut envoyée dans le service de médecine interne où elle a été hospitalisée et mise en exéat après 20 jours de prise en charge pour polysérite tuberculeuse. L'échographie cardiaque réalisée à la sortie objectivait un épanchement péricardique de grande abondance, circonférentiel, de $23,4 \mathrm{~mm}$ et une dilatation de l'aorte ascendante à $63 \mathrm{~mm}$ motivant son transfert en cardiologie. L'examen cardiovasculaire avait retrouvé un souffle d'insuffisance aortique $2 / 6$, un syndrome d'insuffisance cardiaque droite et un syndrome anémique avec de bons chiffres tensionnels à138/22 mmHg. L'électrocardiogramme s'inscrivait en rythme sinusal avec une tachycardie régulière à $115 / \mathrm{mn}$, des anomalies de la repolarisation en circonférentiel à type d'ondes T négatives et aplaties. Le diagnostic de dissection aortique ne faisant pas de doute, la patiente était mise aussitôt sous aténolol avec maintien de la TA autour de 120/80 mmHg. Douze (12) heures après son admission, elle était décédée dans un tableau clinique de choc hypovolémique.

\section{Cas 6}

Il s'agit d'un homme noir âgé de 65 ans, hypertendu connu depuis 15 ans avec hypercholestérolémie. Il a été opéré en 2010 pour une dissection aortique de type A étendue à l'aorte abdominale viscérale et aux artères iliaques droites, par mise en place d'un tube sus-coronaire (Prothèse Dacron LPI N॰28). Il existait une dissection résiduelle au-delà du tube aortique. Il a été réopéré en 2011 pour une dilatation anévrysmale à $71 \mathrm{~mm}$ du sinus de Valsalva, par une intervention de type Bentall avec un tube de Dacron valvé par une valve mécanique (CARBOMEDICS $25 \mathrm{~mm}$ ) avec persistance d'un pseudo-anévrysme sur dissection de l'aorte thoracique descendante $(51 \mathrm{~mm}$ en décembre 2014, 60mm en mai 2017 et 66mm en 2017). Il était admis en hospitalisation de cardiologie pour suspicion de dissection de l'aorte. L'interrogatoire retrouvait un début remontant à $48 \mathrm{~h}$ avant son admission marqué par une douleur de la base postérieure du cou, de repos à irradiation descendante le long du rachis et vers les lombes, survenant par épisodes de 10 
minutes, d'intensité 6/10, intercalés par un fond douloureux permanent. Cette douleur était accompagnée d'une asthénie importante et n'avait pas donné lieu à une médication particulière. L'apparition d'un épisode douloureux rétrosternale plus intense à 8/10, accompagné de nausées, de vomissement et de sueurs profuses motiva l'hospitalisation. A l'admission, l'examen clinique objectivait : Poids $=88 \mathrm{~kg}$; tour de taille $=108 \mathrm{~cm} ;$ Taille $=1 \mathrm{~m} 70 ; \mathrm{IMC}=30$ $\mathrm{kg} / \mathrm{m}^{2} ; \mathrm{TA}=114 / 72 \mathrm{~mm} \mathrm{Hg}$, un syndrome d'épanchement pleural gauche. L'ECG retrouvait un rythme sinusal régulier à $60 \mathrm{bpm}$, une ischémie sousépicardique inférieure, une hypertrophie atrioventriculaire gauche, un bloc de branche droit complet et un hémibloc antérieur. La radiographie thoracique mettait en évidence un élargissement du médiastin avec un émoussement du cul-de-sac costodiaphragmatique gauche. L'échographie Doppler cardiaque retrouvait une bonne perméabilité de la valve mécanique aortique avec gradient moyen VG-AO à $12 \mathrm{mmHg}$ et anévrysme de l'aorte abdominale à $48 \mathrm{~mm}$. L'angioscanner thoracique mettait en évidence une dissection aortique de type $\mathrm{B}$ étendue jusqu'à l'artère iliaque gauche sur dilatation anévrismale de l'aorte post isthmique mesurée à 70x80 mm, avec hémothorax bilatéral prédominant à gauche, collapsus pulmonaire gauche; un retro-hémopéritoine. L'hémodynamie initialement bonne s'est dégradée avec la survenue d'un choc hypovolémique qui a nécessité son transfert en réanimation où il a bénéficié d'un traitement par transfusion de sang total, morphine, assistance respiratoire. Le patient est transféré par avion sanitaire au bloc opératoire de chirurgie cardiovasculaire de 1'hôpital Européen Georges Pompidou (Paris) et décèdera quelques heures après sa sortie du bloc.

\section{Discussion}

La DA représente 1'un des plus grands drames cardiologiques rares dont la forme typique est révélée par la triade douleur thoracique insuffisance aortique (IAo) - abolition d'un pouls.

\section{Symptômes révélateurs, circonstances de découvertes, signes}

Parmi les 6 cas rapportés, 5 ont présenté une douleur thoracique d'ancienneté variable et quatre ont eu un souffle d'IAo. Parfois la clinique peut être trompeuse, soit parce que le siège ou le type de douleur sont atypiques, soit parce qu'une complication est au premier rang rendant ainsi le diagnostic fortuit. La douleur thoracique était typique, rétrosternale migratrice chez 2 patients. La douleur était toutefois absente dans l'histoire clinique du cas $\mathrm{N}^{\circ} 2$. Elle serait absente ou n'est pas signalée par le patient dans 5 à $15 \%$ des cas (Veyssier-Belot, 1999).

Chez trois de nos patients, la découverte de la DA a été fortuite. C'est le cas de la première patiente qui en dehors de la DA présentait une embolie pulmonaire, motif de réalisation de la TDM (Tomodensitométrie). Cette 
embolie pourrait être une complication de la DA par la compression médiastinale de l'artère pulmonaire. L'observation d'un patient présentant une dissection de type $\mathrm{B}$ associée à une embolie pulmonaire a été rapportée dans la littérature (Ndobo et al., 2016). Le cas N² quant à lui présentait une bronchopathie dont l'exploration a abouti au diagnostic de DA. La DA a été découverte chez le cas $\mathrm{N}^{\circ} 5$ lors de l'exploration des complications réputées être de mauvais pronostic : hémopericarde, l'hémothorax et hémopéritoine. Selon la littérature, l'insuffisance aortique est la complication la plus fréquente (40-70\%) de la DA de type A de Stanford (Veyssier-Belot, 1999). L'insuffisance aortique a été retrouvée chez quatre de nos patients.

Ces cas cliniques partagent des particularités diagnostiques propres au contexte de $1^{\prime}$ Afrique sub-saharienne. Il s'agit notamment du recours tardif aux soins, parfois plusieurs semaines après la douleur initiale. L'impact des considérations culturelles africaines et l'ignorance des patients par rapport à l'importance des symptômes, notamment de la douleur thoracique, sont entre autres les explications de ce recours tardif aux soins. Ainsi, le diagnostic de la DA est souvent fait au stade des complications, avec une symptomatologie parfois atypique.

\section{Facteurs étiologiques}

L'hypertension artérielle (HTA) a été retrouvée comme antécédent chez 5 patients dont 3 avaient au moment de l'admission une HTA de grade 3 selon la classification de l'OMS. Deux patients avaient une différentielle de + $20 \mathrm{mmHg}$ de pression artérielle entre les membres thoraciques. L'HTA est selon la littérature le facteur étiologique le plus fréquent et elle a été rapportée dans les séries africaines (Sarr et al., 2004; Ndobo et al., 2016; Bouramoué et al., 2001).

Il y avait 4 femmes et 2 hommes, âgés en moyenne de 53,33 ans (3365ans). Quatre patients sur 6 étaient âgés d'au moins 50 ans ,étant donc en conformité avec la littérature qui identifie l'âge mûr comme facteur d'altération de la résistance de la paroi aortique.

L'absence de facteurs de risque cardiovasculaire, le jeune âge et la survenue d'une vive douleur thoracique pendant l'accouchement chez le cas $\mathrm{N}^{\circ} 1$ nous ont amené à évoquer une DA survenue en peripartum. Cette dernière période est particulièrement à risque, puisque le débit cardiaque est augmenté de $65 \%$ après l'expulsion (Barrett et al., 1982) et la localisation la plus fréquente est l'aorte ascendante. Pour certains auteurs, $50 \%$ des dissections aortiques chez la femme de moins de 40 ans apparaissent pendant la grossesse, en particulier au cours du troisième mois de la grossesse ou durant le postpartum (Haberstich et al., 2001). Ceci n'est pas admis par d'autres (Oskoui et al., 1994). Cependant, des facteurs de risque spécifiques à la grossesse ont été retenus par la majorité : les facteurs hémodynamiques tels que l'augmentation 
de la volémie et du débit cardiaque, et la compression de l'aorte et des vaisseaux iliaques par l'utérus gravide ; les modifications histologiques et biochimiques de la paroi des vaisseaux qui semblent d'origine hormonale, et plus en rapport avec les estrogènes circulants qu'avec la progestérone : hypoplasie de l'intima, épaississement de la média avec fragmentation des fibres réticulées (Jacques et al., 2012).

\section{Examens complémentaires}

La suspicion d'une DA est aisée lorsque l'ECG est normal dans un contexte de douleur thoracique. Mais souvent l'ECG est anormal et trompeur, mettant en évidence des anomalies de la repolarisation (cas 3, 4 et 5).

Dans nos pays à plateau technique limité, la radiographie a toujours son intérêt et reste non négligeable dans la démarche diagnostique. Selon la littérature, elle est anormale dans $80 \%$ des cas et met en évidence un élargissement du médiastin supérieur avec dilatation du bouton aortique, un épanchement pleural gauche (Hartnell et al., 1993). La radiographie thoracique était contributive au diagnostic dans les 4 cas où elle a été prescrite, l'échographie Döppler cardiaque l'était chez 5 patients alors que l'ECG n'a été d'aucune contribution au diagnostic.

L'élargissement du médiastin, signe radiologique retrouvé chez les patients, était le principal incitateur à la réalisation de la tomodensitométrie thoracique, outil essentiel dont tous nos patients ont bénéficié.

Nous avons observé une DA de type A de Stanford chez 5 patients, et ceci est en accord avec la littérature qui identifie le type A comme le plus fréquent (Veyssier-Belot, 1999). Chez le cas clinique 6, il s'agissait d'un nouvel épisode de dissection aortique de type $\mathrm{B}$ chez un patient déjà opéré pour dissection aortique de type A et porteur d'un Bentall.

\section{Traitement et évolution}

Tous nos patients ont bénéficié d'un traitement médical. Quelque soit le type de DA, un traitement antalgique et le contrôle de la pression artérielle sont recommandés. En dehors du traitement médical, schématiquement, pour les atteintes de l'aorte ascendante, une intervention chirurgicale en urgence reste la règle, parfois associée à un geste endovasculaire, en particulier en cas de malperfusion viscérale (Rousseau et al., 2009).

L'évolution naturelle de la dissection de type A est catastrophique, avec un taux de mortalité de 1 à $2 \%$ par heure après l'apparition du premier symptôme. Quatre des cinq patients ayant une dissection de type A sont décédés par les complications évolutives. Le patient du cas 6 est décédé en post opératoire de la dissection de type B. 
Les patients rescapés reçus dans les hôpitaux pour dissection aortique ont un pronostic effroyable du fait de l'inexistence d'un centre de chirurgie cardiovasculaire pour apporter une réponse thérapeutique appropriée.

\section{Conclusion}

La dissection aortique est de plus en plus fréquente en Afrique grâce à l'acquisition des moyens performants de diagnostic. Le recours tardif aux soins caractérisent les patients dont la symptomatologie est parfois atypique. Le diagnostic est souvent fait au stade de complications. Les facteurs favorisants restent conformes aux données de la littérature présentant comme première étiologie l'hypertension artérielle. La limitation du plateau technique dans nos pays fait payer aux patients un lourd tribut. L'urgence est donc la prévention de cette affection à travers la prise en charge correcte de l'hypertension artérielle.

\section{References:}

1. Barrett, JM., Van Hooydonk, JE., \& Boehm, FH. (1982). Pregnancyrelated rupture of arterial aneurysms. Obsrer Gynecol Sure;37:557667

2. Bouramoué, C., Kimbally-Kaky, G., Nkoua, JL., Le Feuvre, C., Ekoba, J., \& Vacheron, A. (2001). La dissection aortique chez les Noirs : à propos de six cas congolais. Annales de cardiologie et d'angéiologie; 50 (3):133-141.

3. Haberstich, R., Calmelet, P., Charpentier, A., Raiga, J., Schaeffer, R., Treisser, A., \& Brettes, JP. (2001). Multidisciplinary management of aortic dissection complicating pregnancy. J Gynecol Obstet Biol Reprod (Paris); 30(2): 183-6.

4. Hartnell, GG., Wakeley, CJ., Tottle, A., Papouchado, M., \& Wilde, RP. (1993). Limitations of chest radiography in discriminating between aortic dissection and myocardial infarction: implications for thrombolysis. J Thorac Imaging;8(2):152-5.

5. Jacques, V., Guerci, P., Vial, F., Abel, F., \& Bouaziz, H. (2012). Dissection de l'aorte descendante et prééclampsie à 30 semaines d'aménorrhée : prise en charge médicale et césarienne. Annales Françaises d'Anesthésie et de Réanimation;31:67-71.

6. Ndobo, JVD., Diack, B., Mbaye, A., Diagne, D., Kane, A., \& Kane, M. (2016). Dissection aortique aigue type B de Stanford compliquée d'ischémies rénale et des membres inférieurs associée à une embolie pulmonaire. Cardiologie tropicale; $\mathrm{N}^{\circ} 143$.

7. Oskoui, R. \& Lindsay, JJr. (1994). Aortic dissection in women $<40$ years of age and the unimportance of pregnancy. Am J Cardiol; 73(11):821-3. 
8. Roudaut, R., Lafitte, S., Durrieu-Jaïs, C., Réant, P., Mignot, A., Laurent, F., \& Labrousse, L. (2009). Dissection de l'aorte thoracique. Encycl Méd Chir (Editions Scientifiques et Médicales Elsevier SAS, Paris, tous droits réservés), Cardiologie, 11-650-A-10, 14p.

9. Rousseau, H., Chabbert, V., Marcheix, B., El Hassar, O., Cron, C., \& Lopez, S. (2009). Les syndromes aortiques aigus. STV; 21(3):112-25

10. Sarr, M., Doucoure, I., Dao, M., Diop, IB., BA S A., \& Diouf, SM. (2004). Etude clinique et évolutive de 13 cas de dissection aortique. Cardiologie tropicale; $\mathrm{N}^{\circ} 117$.

11. Veyssier-Belot, C. (1999). Dissections aortiques. Encycl Méd Chir (Editions Scientifiques et Médicales Elsevier SAS, Paris, tous droits réservés), 11-650-A-10, 5p. 